\title{
Confirmation of anti-DFS70 antibodies is needed in routine clinical samples with DFS staining pattern
}

\author{
ESVET MUTLU, METE EYIGÖR, DERYA MUTLU, MERAL GÜLTEKIN
}

Department of Medical Microbiology, Division of Immunology, Akdeniz University School of Medicine, Antalya, Turkey

\begin{abstract}
Background: Recognition of nuclear dense fine speckled (DFS) pattern by indirect immunofluorescence (IIF) is not easy. Thus, confirming the presence of these antibodies might be needed. In this study, we aimed to determine the frequency of DFS pattern in our diagnostic laboratory and to investigate the presence of anti-DFS70 antibodies in samples showing DFS pattern by two commercially available research kits retrospectively.

Material and methods: Seventy-four sequential serum samples with DFS pattern on HEp2010 cell substrates by IIF were included in this study. The semiquantitative DFS70 ELISA Kit (MBL International Corporation, Woburn, UK) was used for detection of anti-DFS70 antibodies in these samples. Twenty selected samples were tested for the presence of anti-DFS70 antibodies using ANA Line Immunoassay (LIA) (Immco Diagnostics, New York, USA).

Results: Sixty-two (83.8\%) of 74 serum samples were found positive with ELISA, when $15 \mathrm{U} / \mathrm{ml}$ was taken as a reference value. Among 18 samples that were found positive by ELISA, five were negative for anti-DFS70 antibodies by LIA, while 13 were found positive. The lowest ELISA result of the sample that was positive by LIA was found to be $45.3 \mathrm{U} / \mathrm{ml}$. When $45.3 \mathrm{U} / \mathrm{ml}$ was considered as a reference value, 45 (60.8\%) of 74 serum samples were positive by ELISA. Nineteen of 20 patients had no SARD, while one had systemic lupus erythematosus (SLE).

Conclusions: DFS pattern should be confirmed with an objective method such as ELISA, LIA, or IB. We think that confirmation tests for detection of anti-DFS70 antibodies should be included in diagnostic algorithms.
\end{abstract}

Key words: DFS70, indirect immunofluorescence, line immunoassay, systemic autoimmune rheumatic disease.

(Cent Eur J Immunol 2016; 41 (1): 6-11)

\section{Introduction}

The indirect immunofluorescence (IIF) assay is one of the most commonly used methods and is recommended as the "gold standard" for screening of antinuclear antibodies (ANA) [1].

The typical nuclear dense fine speckled (DFS) pattern on HEp2 cells is recognised as uniformly distributed fine speckles throughout the interphase nucleus and on metaphase chromatin, and it is considered as a standard pattern according to the pattern standardisation initiative [2,3]. Since a 70-kd protein was recognised on immunoblotting (IB), the antigen was initially termed dense fine speckles 70 and was later identified as lens epithelium-derived growth factor (LEDGF) or DNA binding transcription coactivator p75 [4, 5].

Anti-DFS70 antibodies were initially identified in a patient with interstitial cystitis [6]. Since their first de- scription, anti-DFS70 antibodies have been associated with a variety of chronic inflammatory diseases and cancer [7-9]. The highest prevalence of these antibodies was reported in patients with Vogt-Harada syndrome $(66.7 \%)$, atopic dermatitis $(30 \%)$, and asthma $(16 \%)[3,6,10$, 11]. On the other hand, several studies showed that anti-DFS70 antibodies were common among ANA-positive healthy individuals, while they were found to be rare in patients with systemic autoimmune rheumatic diseases (SARD) [12-15].

Although IIF technique has been recommended for ANA screening by the study group of the American College of Rheumatology (ACR), it lacks universal standardisation and leads to false positive results [1]. Recognition of DFS pattern by IIF is not easy and there might be difficulties in discrimination of this pattern from the quasihomo-

Correspondence: Esvet Mutlu, Department of Medical Microbiology, Division of Immunology, Akdeniz University School of Medicine, Dumlupinar Bulvari, 07070 Antalya, Turkey, e-mail: dresvetmutlu@yahoo.com Submitted: 28.07.2015; Accepted: 23.02.2016 
geneous pattern defined by Mariz et al. [12]. On the other hand, the presence of the DFS pattern is important because it is considered as strong evidence against a diagnosis of SARD [13, 15-17]. The possibility of false-positive results seems to be high in diagnostic laboratories [18]. Therefore, the presence of anti-DFS70 antibodies should be confirmed by a more objective method.

The aims of our study were to determine the frequency of DFS pattern in our diagnostic laboratory setting and to investigate the presence of anti-DFS70 antibodies in samples showing a DFS pattern by two commercially available research kits, retrospectively.

\section{Material and methods}

A total of 1786 serum samples, which were submitted for ANA screening from different clinics, were analysed retrospectively. Among them, 74 samples showing DFS pattern on HEp2010 cell substrates by IIF were chosen and included in this study. Sixty of the patients $(81.1 \%)$ were women, while 14 were men (18.9\%). Samples with DFS pattern were identified using Mosaic HEp-2010/Liver (Monkey) cell substrate (Euroimmun; Germany). Sera were screened at $1: 100$ dilution. This study was approved by the local Ethical Committee of Akdeniz University.

Previously frozen and stored samples at $-80^{\circ} \mathrm{C}$ were thawed and studied retrospectively. The semiquantitative DFS70 Enzyme-linked immunosorbent assay (ELISA) kit (MBL International Corporation, Woburn, UK) was used for detection of anti-DFS70 antibodies in human serum. ELISA was performed according to the manufacturer's instructions. The reference value given by the kit was $15 \mathrm{U} / \mathrm{ml}$.

We selected 20 samples according to their ELISA results (Unit/ml) in an ascending order within a range of $0 \mathrm{U} / \mathrm{ml}$ (the lowest) and $128.9 \mathrm{U} / \mathrm{ml}$ (the highest) (two samples with results under $15 \mathrm{U} / \mathrm{ml}$, seven with results between 22.4-48.1 U/ml, and 11 with results $\geq 50 \mathrm{U} / \mathrm{ml}$ ). Anti-DFS70 antibodies of these samples were tested using ANA Line Immunoassay (LIA) (Immco Diagnostics, New York, USA) according to the manufacturer's instructions. Briefly, strips were incubated with patient serum. Strips were washed according to the protocol and ready-to-use conjugate was added to the strips. After incubation and wash steps, the ready-to-use substrate was added to the strips and strips were incubated for $10 \mathrm{~min}$ utes. During incubation three visible lines occurred for serum, conjugate, and cut-off control lines. Reactions were read visually according to the cut-off line. ANA LIA strips included PM-Scl 100, PM-Scl 75, SSA/Ro52, SSA/Ro-60, Jo1, Ribosomal Protein P, Nucleosomes, DNA, Histones, Sm, U1 SnRNP6B, U1 SnRNP A, U1 SnRNP C, SSB/La, Scl 70, CENP-B, and PCNA antigens in addition to DFS70. Clinical diagnoses of patients were analysed retrospectively by clinical chart review of medical records.

\section{Results}

Among the 1786 samples that were submitted for ANA testing $368(20.6 \%)$ were ANA positive by IIF. Ninety samples had DFS pattern (24.5\% of ANA positive samples and $5 \%$ of all samples tested for ANA). We included 74 of 90 samples in the study, as 16 patients remained undiagnosed during the study or we were not able to obtain their diagnostic information. Among the 74 patients who had DFS pattern, $68(91.9 \%)$ did not have any SARD, while six $(8.1 \%)$ of these patients were diagnosed with SARD. Three patients had systemic lupus erythematosus (SLE), two had Sjögren's syndrome, and one had scleroderma. Additionally, six patients had rheumatoid arthritis (RA) while two patients were diagnosed with familial Mediterranean fever (FMF).

Anti-DFS70 antibodies of 74 samples were tested by ELISA and the results changed between 0 and $188.8 \mathrm{U} / \mathrm{ml}$ (Table 1). When $15 \mathrm{U} / \mathrm{ml}$ was taken as a reference value, as recommended by the manufacturer, 62 (83.8\%) of 74 serum samples were found positive with MBL ELISA Kit. Fifty-seven of the patients $(91.9 \%)$ who were found positive with MBL ELISA did not have any SARD, while five $(8.1 \%)$ of them were SARD. One of the $12(8.3 \%)$ patients who were negative with ELISA had SARD (SLE).

Among 20 samples tested by LIA, seven were negative for anti-DFS70 antibodies, while 13 were found positive. ELISA results of 20 serum samples were between 0 and $128.9 \mathrm{U} / \mathrm{ml}$. There were only two samples under the cut-off value (with values 0 and $7.1 \mathrm{U} / \mathrm{ml}$ ); the rest of the samples had ELISA values higher than $15 \mathrm{U} / \mathrm{ml}$. ELISA results of seven LIA-negative samples were between 0 and 43.7 U/ml. The lowest ELISA result of the sample that was positive by LIA was $45.3 \mathrm{U} / \mathrm{ml}$. Among 74 samples, $45(60.8 \%)$ had ELISA results of $45.3 \mathrm{U} / \mathrm{ml}$ and above. Median value of ELISA in LIA-positive samples was 76 (range 45.3-128.9, $n=13$ ) and median value of negative samples was 28.3 (range $0-43.7, n=7$ ). The clinical characteristics of patients and their LIA and ELISA results are shown in Table 2.

According to the medical records, 19 of 20 patients had no diagnosis of SARD. One patient was diagnosed as SLE. Additionally, two patients had FMF.

\section{Discussion}

Typical DFS pattern by IIF was described as dense fine speckled staining of both interphase nuclei and metaphase chromatins. It was characterised by heterogeneity in the brightness and size of the speckles. Another pattern which might resemble DFS pattern, referred to as quasihomogeneous pattern by Mariz et al., was defined as fine, grainy staining of both nuclei and metaphase chromatins. It was underlined that recognition of DFS pattern and discrimination from the quasihomogeneous pattern especially at low 
Table 1. ELISA results of patients $(n=74)$

\begin{tabular}{|c|c|c|c|c|c|c|c|}
\hline Patient no. & $\begin{array}{c}\text { ELISA result } \\
(\mathbf{U} / \mathbf{m l})\end{array}$ & Patient no. & $\begin{array}{c}\text { ELISA result } \\
(\mathrm{U} / \mathrm{ml})\end{array}$ & Patient no. & $\begin{array}{c}\text { ELISA result } \\
(\mathbf{U} / \mathbf{m l})\end{array}$ & Patient no. & $\begin{array}{c}\text { ELISA result } \\
\text { (U/ml) }\end{array}$ \\
\hline 1 & 92.3 & 24 & 1.4 & 47 & 28.3 & 69 & 0 \\
\hline 3 & 0 & 25 & 0 & 48 & 105.5 & 70 & 81.8 \\
\hline 4 & 7.1 & 26 & 137.2 & 49 & 28.3 & 71 & 36.2 \\
\hline 5 & 1.3 & 28 & 86 & 50 & 0 & 72 & 148.3 \\
\hline 6 & 90.3 & 29 & 0 & 51 & 60.0 & 73 & 29.4 \\
\hline 8 & 188.8 & 30 & 36.9 & 52 & 88.0 & 74 & 130 \\
\hline 9 & 99.9 & 31 & 47.4 & 54 & 3.9 & 75 & 43.7 \\
\hline 10 & 128.9 & 32 & 129.8 & 55 & 22.4 & 76 & 29.2 \\
\hline 12 & 140.1 & 33 & 116.5 & 56 & 161.1 & 77 & 65.7 \\
\hline 13 & 68.2 & 34 & 151.1 & 57 & 59 & 78 & 74.6 \\
\hline 14 & 50.0 & 35 & 6.9 & 58 & 20.2 & 79 & 36.1 \\
\hline 15 & 57.3 & 39 & 12.8 & 59 & 158.3 & 80 & 117 \\
\hline 17 & 0 & 40 & 158.7 & 61 & 85.8 & 82 & 23.7 \\
\hline 18 & 75.8 & 41 & 89.0 & 62 & 119 & 83 & 139.9 \\
\hline 19 & 182.2 & 42 & 64.4 & 63 & 35.6 & 84 & 124.1 \\
\hline 20 & 173.1 & 43 & 26.7 & 64 & 43.2 & 85 & 34.3 \\
\hline 21 & 24.8 & 44 & 79.5 & 65 & 45.3 & 90 & 63.6 \\
\hline 22 & 60.1 & 45 & 119.4 & 67 & 48.1 & & \\
\hline 23 & 125.7 & 46 & 73.6 & 68 & 22 & & \\
\hline
\end{tabular}

titres, even by the experienced examiners, was subjective and difficult $[2,19-21]$. As it was reported that DFS pattern tended to occur mostly in healthy individuals while quasihomogeneous pattern was more commonly found in patients with SARD, it is important to diagnose this pattern correctly [12, 13, 16, 17]. Bizzaro et al. suggested that the possibility of false positive results was very high [18]. The prevalence of the DFS pattern among healthy individuals was reported to be high, and this might lead to misdiagnosis in the case of inaccurate determination of the pattern [12]. The importance of confirmation of DFS pattern by using a more objective method such as ELISA or IB was underlined in recent studies [2, 18, 19, 21]. Among 74 patients who had DFS pattern by IIF method in our study, we detected anti-DFS70 antibodies in $62(83.8 \%)$ and $45(60.8 \%)$ patients by ELISA using $15 \mathrm{U} / \mathrm{ml}$ and $45.3 \mathrm{U} / \mathrm{ml}$ as reference values, respectively. The main reason underlying this low concordance might be caused by errors in identification of the pattern and discrimination of it from that of the quasihomogeneous pattern because $74 \%$ $(n=55)$ of our samples were positive at low titre $(1 / 100)$. Therefore, we suggest that it is necessary to standardise the interpretation of ANA between laboratories and confirm the presence of anti-DFS70 antibodies by an objective method.

Previously, sera from selected groups of patients such as healthy individuals and patients with SARD were tested for DFS pattern $[12,14,15]$. In our study, we investigated the frequency of DFS pattern by IIF on Hep-2010 cells in non-selected serum samples submitted for ANA testing in our laboratory. We found that $5 \%$ of our samples showed DFS pattern by IIF. Some studies have reported the frequencies of DFS pattern in clinical samples tested during routine workup as $0.8,1.62$, and $16.5 \%$ [16, 17, 22]. One possible explanation for the differences in the results obtained from these four studies might be due to errors in identification of DFS pattern because it is highly subjective. Representative IIF images of samples showing DFS pattern with positive ELISA and LIA results and negative ELISA and LIA results from our study are shown in Figure 1. Previously it was reported that four different Hep-2 cell lines from different manufacturers gave different results [18]. Bizzaro et al. reported the lowest frequency. In their study, DFS pattern was identified by IIF on Hep- 
Table 2. Clinical information and LIA and ELISA results of patients $(n=20)$

\begin{tabular}{|c|c|c|c|c|c|c|c|}
\hline Patient No & Gender & Diagnosis & Age & $\begin{array}{l}\text { LIA (other } \\
\text { antigens) }\end{array}$ & ANA titre & LIA (DFS70) & DFS70 ELISA \\
\hline 1 & $\mathrm{~F}$ & no SARD & 46 & negative & $1 / 100$ & positive & 92.3 \\
\hline 2 & $\mathrm{~F}$ & no SARD & 10 & negative & $1 / 100$ & negative & 0 \\
\hline 3 & M & no SARD & 12 & negative & $1 / 100$ & negative & 7.1 \\
\hline 4 & $\mathrm{~F}$ & SLE & 30 & histone positive & $1 / 1000$ & positive & 128.9 \\
\hline 5 & M & no SARD & 64 & negative & $1 / 100$ & positive & 68.2 \\
\hline 6 & $\mathrm{~F}$ & RA & 49 & negative & $1 / 1000$ & positive & 50 \\
\hline 7 & $\mathrm{~F}$ & no SARD & 33 & negative & $1 / 100$ & positive & 57.3 \\
\hline 8 & $\mathrm{~F}$ & FMF & 26 & negative & $1 / 100$ & positive & 105.5 \\
\hline 9 & $\mathrm{~F}$ & no SARD & 29 & negative & $1 / 320$ & negative & 28.3 \\
\hline 10 & $\mathrm{~F}$ & FMF & 22 & negative & $1 / 1000$ & positive & 60 \\
\hline 11 & $\mathrm{~F}$ & no SARD & 47 & negative & $1 / 1000$ & positive & 88 \\
\hline 12 & $\mathrm{~F}$ & no SARD & 53 & negative & $1 / 100$ & negative & 22.4 \\
\hline 13 & $\mathrm{~F}$ & no SARD & 39 & negative & $1 / 100$ & positive & 85.8 \\
\hline 14 & M & no SARD & 16 & negative & $1 / 100$ & positive & 119 \\
\hline 15 & M & no SARD & 6 & negative & $1 / 100$ & negative & 43.2 \\
\hline 16 & F & no SARD & 35 & negative & $1 / 1000$ & positive & 45.3 \\
\hline 17 & $\mathrm{~F}$ & RA & 73 & $\begin{array}{l}\text { histone grey } \\
\text { zone }\end{array}$ & $1 / 100$ & positive & 48.1 \\
\hline 18 & M & no SARD & 8 & $\begin{array}{l}\text { PMScl100 } \\
\text { positive }\end{array}$ & $1 / 100$ & negative & 43.7 \\
\hline 19 & F & no SARD & 53 & negative & $1 / 320$ & negative & 34.3 \\
\hline 20 & $\mathrm{~F}$ & no SARD & 46 & negative & $1 / 1000$ & positive & 76 \\
\hline
\end{tabular}

F-female; $M$ - male; SARD - systemic autoimmune rheumatic disease; SLE - systemic lupus erythematosus; RA - rheumatoid arthritis; FMF-familial Mediterranean fever; LIA - line immunoassay
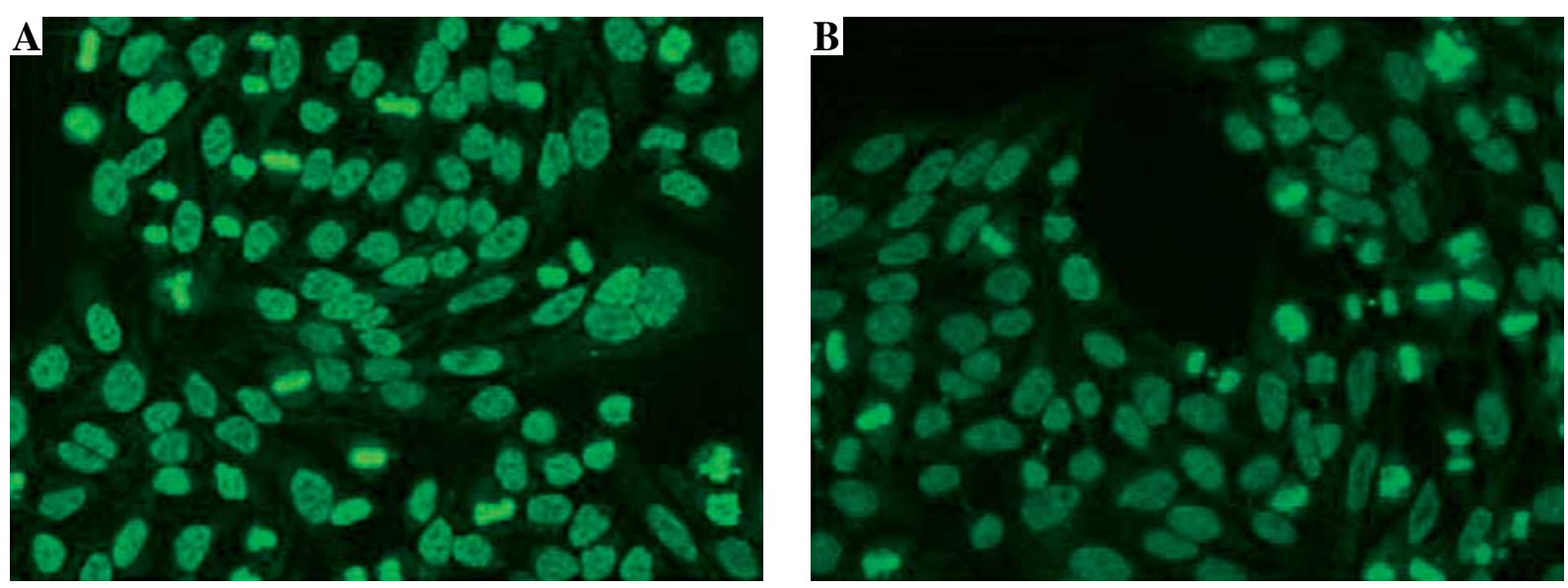

Fig. 1. Indirect immunofluorescence images of two samples with DFS pattern: A) positive with ELISA and LIA, B) negative with ELISA and LIA 
2 cells from 21,516 sera in seven different laboratories, and each centre used its own testing kit [22]. This study lacked inter-laboratory standardisation. In the study, by which the highest frequency was reported, 3263 sera were screened, both on commercial and on custom-made Hep-2 cell slides at $1: 80$ dilution, and analysed by two independent observers. The commercial kit they used in this study was Kallestad from BioRad while we used Hep-2010 cells from Euroimmun [17]. We found slightly higher frequency of DFS pattern than reported by Dellavance $e t$ al., who screened 30,728 serum samples at $1: 80$ dilution [16]. The same commercial kit as ours was used in this study. There might be variations among different ethnic groups or populations in different countries, which might be the cause of differences in prevalences. In light of all these data, we suggest that test procedures, cellular substrates used, and assessment of patterns should be standardised among laboratories.

We used an ELISA kit from MBL, which was intended for research use only. The antigen used in this kit was identical to LEDGF. The cut-off value of ELISA was determined by calculating the average values +3 SD (Standard Deviation) of 50 people with anti-DFS70-negative results by both IIF and IB assay as $15 \mathrm{U} / \mathrm{ml}$. Mahler et al. used this kit previously and detected anti-DFS70 antibodies in 53 of 53 samples with DFS pattern [17]. In our study, we detected anti-DFS70 antibodies in 62 of $74(83.8 \%)$ patients with DFS pattern by this kit. Additionally, we used an LIA assay that was intended for evaluation purposes only. Anti-DFS70 antibodies were negative in seven of the 20 samples by LIA. Among these seven samples, the results of two were below $15 \mathrm{U} / \mathrm{ml}$, while five samples' values were between 22.4 and $43.7 \mathrm{U} / \mathrm{ml}$. The lowest ELISA result of the sample that was anti-DFS70 positive by LIA was found to be $45.3 \mathrm{U} / \mathrm{ml}$. When we took $45.3 \mathrm{U} / \mathrm{ml}$ as reference value the number of anti-DFS70 positive samples declined from 62 to 45 . The discrepancy between ELISA and LIA tests might be due to differences in antigenic sources of ELISA and LIA kits used in this study. It is also plausible that the sensitivity of the LIA assay might be lower than that of ELISA. LIA was defined as a second-generation dot-blot assay [23]. Although reported to lack sensitivity and specificity for certain autoantibodies, it was used for the confirmation of autoantibodies [24-26]. The median value of ELISA in LIA-positive samples was higher than that of the negative samples. It can be speculated that lack of sensitivity of the LIA might be related to the titre rather than antigen presentation.

When groups of patients with SARD were investigated for the presence of anti-DFS70 antibodies, the prevalence was determined to be low $(0 \%, 4.4 \%$, and $1.5 \%)$ in several studies $[12,14,15]$. The prevalences obtained were quite different among samples sent to the routine clinical diagnostic laboratory. It was reported that $2.8 \%, 6.1 \%$, $13.4 \%$, and $39 \%$ of patients with anti-DFS70 antibodies had SARD in several studies [13, 16, 17, 27]. In our study, six $(8.1 \%)$ of 74 patients who had DFS70 staining pattern were diagnosed with SARD. Although we did not investigate the presence of anti-DFS70 antibodies by LIA in all of our patients, these numbers seem to represent the approximate percentage of patients with anti-DFS70 antibodies who had SARD. We speculate that the majority of our patients $(91.9 \%)$ with this pattern do not have any SARD, which is consistent with previous reports [12-17]. On the other hand, we found that the prevalence of SARD in DFS70 antibody-positive $(8.1 \%)$ and -negative $(8.3 \%)$ patients was similar. As has been reported previously, the presence of anti-DFS70 antibodies does not always rule out SARD [28]. Therefore, detection of anti-DFS70 antibodies should be included in test algorithms for ANA testing. New confirmation tests for detection of anti-DFS70 antibodies should be developed, or the tests that are currently being used for determination of ANAs should be improved by the inclusion of anti-DFS70 antibodies. There are a few commercially available kits that are already in use. In addition, it has been reported that a reference serum with anti-DFS70 antibodies will be available by the Autoantibody Standardising Committee [29].

We had data on other autoantibodies of only 20 patients tested for the presence of anti-DFS70 autoantibodies by LIA. In addition to DFS70, one patient with SLE had autoantibodies against histones. One of the two patients who had RA was histone grey zone while the other was PM-Scl100 positive. As a limitation of our study, we did not have any data on other autoantibodies in the remaining 54 patients.

Of interest, two patients with anti-DFS70 antibodies were diagnosed as FMF, a hereditary autoinflammatory disease that is mainly seen in the Mediterranean region $[30,31]$. Although anti-DFS70 antibodies have been associated with a variety of chronic and inflammatory diseases, it has not been linked to FMF [3, 6]. Further research investigating the presence of anti-DFS70 antibodies in patients with FMF might be of value.

In conclusion, because of the subjectivity in recognising the pattern and lack of standardisation, DFS staining pattern should be confirmed by an objective method such as ELISA, LIA, or IB. As the presence of anti-DFS70 antibodies does not always mean the absence of pathology, confirmation of these antibodies should be included in diagnostic algorithms in routine laboratories.

The authors declare no conflict of interest.

\section{References}

1. Meroni PL, Schur PH (2010): ANA screening: an old test with new recommendations. Ann Rheum Dis 69: 1420-1422.

2. Fritzler MJ (2011): The antinuclear antibody test: last or lasting gasp? Arthritis Rheum 63: 19-22. 
3. Ochs RL, Muro Y, Si Y, et al. (2000): Autoantibodies to DFS $70 \mathrm{kd} /$ transcription coactivator p75 in atopic dermatitis and other conditions. J Allergy Clin Immunol 105: 1211-1220.

4. Ge H, Si Y, Roeder RG (1998): Isolation of cDNAs encoding novel transcription coactivators p52 and p75 reveals an alternate regulatory mechanism of transcriptional activation. EMBO J 17: 6723-6729.

5. Shinohara T, Singh DP, Fatma N (2002): LEDGF, a survival factor, activates stress-related genes. Prog Retin Eye Res 21: 341-358.

6. Ochs RL, Stein TW Jr., Peebles CL, et al. (1994): Autoantibodies in interstitial cystitis. J Urol 151: 587-592.

7. Muro Y (2001): Autoantibodies in atopic dermatitis. J Dermatol Sci 25: 171-178.

8. Ganapathy V, Casiano CA (2004): Autoimmunity to the nuclear autoantigen DFS70 (LEDGF): what exactly are the autoantibodies trying to tell us? Arthritis Rheum 50: 684-688.

9. Okamoto M, Ogawa Y, Watanabe A, et al. (2004): Autoantibodies to DFS70/LEDGF are increased in alopecia areata patients. J Autoimmun 23: 257-266.

10. Ayaki M, Ohoguro N, Azuma N, et al. (2002): Detection of cytotoxic anti-LEDGF autoantibodies in atopic dermatitis. Autoimmunity 35: 319-327.

11. Yamada K, Senju S, Shinohara T, et al. (2001): Humoral immune response directed against LEDGF in patients with VKH. Immunol Lett 78: 161-168.

12. Mariz HA, Sato EI, Barbosa SH, et al. (2011): Pattern on the antinuclear antibody-HEp-2 test is a critical parameter for discriminating antinuclear antibody-positive healthy individuals and patients with autoimmune rheumatic diseases. Arthritis Rheum 63: 191-200.

13. Miyara M, Albesa R, Charuel JL, et al. (2013): Clinical phenotypes of patients with anti-DFS70/LEDGF antibodies in a routine ANA referral cohort. Clin Dev Immunol 2013: 703759.

14. Muro Y, Sugiura K, Morita Y, Tomita Y (2008): High concomitance of disease marker autoantibodies in anti-DFS70/ LEDGF autoantibody-positive patients with autoimmune rheumatic disease. Lupus 17: 171-176.

15. Watanabe A, Kodera M, Sugiura K, et al. (2004): Anti-DFS70 antibodies in 597 healthy hospital workers. Arthritis Rheum 50: 892-900.

16. Dellavance A, Viana VS, Leon EP, et al. (2005): The clinical spectrum of antinuclear antibodies associated with the nuclear dense fine speckled immunofluorescence pattern. J Rheumatol 32: 2144-2149.

17. Mahler M, Parker T, Peebles CL, et al. (2012): Anti-DFS70/ LEDGF antibodies are more prevalent in healthy individuals compared to patients with systemic autoimmune rheumatic diseases. J Rheumatol 39: 2104-2110.

18. Bizzaro N, Tonutti E, Villalta D (2011): Recognizing the dense fine speckled/lens epithelium-derived growth factor/ p75 pattern on HEP-2 cells: not an easy task! Comment on the article by Mariz et al. Arthritis Rheum 63: 4036-4037.

19. Mahler M, Hanly JG, Fritzler MJ (2012): Importance of the dense fine speckled pattern on HEp-2 cells and anti-DFS70 antibodies for the diagnosis of systemic autoimmune diseases. Autoimmun Rev 11: 642-645.

20. Mahler M, Fritzler MJ (2012): The clinical significance of the dense fine speckled immunofluorescence pattern on HEp-2 cells for the diagnosis of systemic autoimmune diseases. Clin Dev Immunol 2012: 494356.
21. Basu A, Woods-Burnham L, Ortiz G, et al. (2015): Specificity of antinuclear autoantibodies recognizing the dense fine speckled nuclear pattern: Preferential targeting of DFS70/ LEDGFp75 over its interacting partner MeCP2. Clin Immunol 161: 241-250.

22. Bizzaro N, Tonutti E, Visentini D, et al. (2007): Antibodies to the lens and cornea in anti-DFS70-positive subjects. Ann N Y Acad Sci 1107: 174-183.

23. Mahler M, Meroni PL, Bossuyt X, Fritzler MJ (2014): Current concepts and future directions for the assessment of autoantibodies to cellular antigens referred to as anti-nuclear antibodies. J Immunol Res 2014: 315179.

24. Almeida GD, Cabrera de LA, Rodriguez Perez MC, et al. (2010): Efficiency of different strategies to detect autoantibodies to extractable nuclear antigens. J Immunol Methods 360: 89-95.

25. Sener AG, Afsar I, Demirci M (2014): Evaluation of antinuclear antibodies by indirect immunofluorescence and line immunoassay methods': four years' data from Turkey. APMIS 122: $1167-1170$.

26. Lee SA, Kahng J, Kim Y, et al. (2012): Comparative study of immunofluorescent antinuclear antibody test and line immunoassay detecting 15 specific autoantibodies in patients with systemic rheumatic disease. J Clin Lab Anal 26: 307-314.

27. Fitch-Rogalsky C, Steber W, Mahler M, et al. (2014): Clinical and serological features of patients referred through a rheumatology triage system because of positive antinuclear antibodies. PLoS One 9: e93812.

28. Craig WY, Ledue TB (2011): The dense fine speckled antinuclear antibody pattern in autoimmune rheumatic disease: comment on the article by Mariz et al. Arthritis Rheum 63: 1759 .

29. Sheldon J, Dellavance A (2015): Strategies for building reference standards for autoantibodies. Front Immunol 6: 194.

30. Berkun Y, Eisenstein EM (2014): Diagnostic criteria of familial Mediterranean fever. Autoimmun Rev 13: 388-390.

31. Eisenstein EM, Berkun Y, Ben-Chetrit E (2013): Familial Mediterranean fever: a critical digest of the 2012-2013 literature. Clin Exp Rheumatol 31: 103-107. 